\title{
Clinical and demographic features in Iranian multiple sclerosis patients: a systematic review and meta-analysis
}

\author{
Leily Mahmudi ${ }^{1}$, Moslem Moslemirad ${ }^{2}$, Bafrin Dabestani ${ }^{3}$, Masoumeh Shohani ${ }^{4}$ \& Milad \\ Azami*,5 \\ ${ }^{1}$ School of Medicine, Dezful University of Medical Sciences, Dezful, Iran \\ ${ }^{2}$ School of Nursing \& Midwifery, Yasuj University of Medical Science, Yasuj, Iran \\ ${ }^{3}$ Faculty of Allied Medical Sciences, Shahid Beheshti University of Medical Sciences, Tehran, Iran \\ ${ }^{4}$ Department of Nursing, Faculty of Allied Medical Sciences, Ilam University of Medical Sciences, Ilam, Iran \\ ${ }^{5}$ School of Medicine, Ilam University of Medical Sciences, Ilam, Iran \\ *Author for correspondence: Tel.: +98 912029 1600; Milad98azami@gmail.com
}

Aim: This study was conducted to determine the clinical and demographic features in Iranian multiple sclerosis (MS) patients including clinical courses, positive MS family history, disability and age of disease onset. Materials \& methods: All stages of the study were reported according to the PRISMA guidelines. A systematic review of published literature in Persian and English journals with a comprehensive search was conducted on English international databases including Scopus, PubMed/Medline, Cochrane Library, Science Direct, Web of Science, Embase, PsycINFO, as well as the Google Scholar search engine and national databases. Results: The prevalence of clinical courses of relapsing remitting, primary progressive, secondary progressive and relapsing progressive in Iranian patients with MS was $77.1 \%(95 \% \mathrm{Cl}$ : $72.2-$ 81.3), 6.2\% (95\% Cl: 4.1-9.2), 9.5\% (95\% Cl: 6.8-13.1) and 0.4\% (95\% Cl: 0.2-0.8), respectively. Positive family history of MS in Iranian MS patients was $8.9 \%$ (95\% Cl: 6.9-11.4). The mean age of disease onset was 28.9 years ( $95 \% \mathrm{Cl}: 27.8-30.1$ ). Conclusion: The results of this meta-analysis in an Iranian population can provide useful information for neurologists and healthcare.

First draft submitted: 2 July 2018; Accepted for publication: 10 December 2018; Published online: 24 May 2019

Keywords: age of onset $\bullet$ disability $\bullet$ Iran $\bullet$ meta-analysis $\bullet$ multiple sclerosis $\bullet$ prevalence

Multiple sclerosis (MS) is an inflammatory demyelinating disease of the CNS, in which the protective sheath of the myelin that covers the nerve cells is destroyed and can cause permanent disability in the patient $[1,2]$. The exact pathophysiology of MS is not known, but it seems to be a multifactorial disorder associated with genetic talents and environmental triggers [2].

It is a common neurological disorder in adults and affects about 2.3 million people around the world. The disease is often seen as an active population in people aged 20-40 [3]. Clinical manifestations of MS include opiate neuritis, central paralysis, sensory imbalance, disturbances, cognitive impairment, fatigue and sleep disorders. For this disease, four clinical forms have been defined: relapsing remitting (RR), primary progressive (PP), secondary progressive (SP) and relapsing progressive (RP) [4,5]. Iran is a Muslim country in the Middle East and latitude of $32^{\circ} 00$ and longitude of $53^{\circ} 00$ and has 31 provinces. There are various ethnic groups, including Fars, Kurds, Mazani, Gilak, Lor, Turks, Arabs and Baluchis, and are now united under Iranian culture. Based on the 2013 Atlas of the MS, the Middle Eastern and North African countries are in the low to moderate danger zone [6]. However, recent studies report a moderate to high MS incidence in these areas, especially among women [7-11].

According to Kurtzke based on geographical areas, Iran is in a low prevalence region of MS (0-4 per 100,000 people) $[12,13]$. But according to a recent meta-analysis (2019), its incidence and prevalence in Iran was estimated 3.4 per 100,000 and 29.3 per 100,000 people, respectively. Over time, the incidence and prevalence has increased significantly [14]. 
In Iran, several epidemiological studies have been conducted among MS patients, and so far no meta-analytical studies have been conducted among them [15-18]. In systematic review and meta-analysis, a comprehensive picture of the dimensions of a problem in society can be found in the process by examining all the documents and combining them [19-21]. Therefore, the present study was conducted to evaluate the clinical and demographic features in Iranian MS patients including clinical courses, positive MS family history, disability and age of disease onset.

\section{Materials \& methods Study protocol}

The stages of the study include the search, selection of studies, quality assessment, data extraction and statistical analysis were reported according to the PRISMA guidelines for systematic review and meta-analysis [21].

\section{Search strategy}

A systematic review of published literature in Persian and English journals with a comprehensive search was conducted on six national databases including Barakat Knowledge Network System (http://health.barakatkns.com), Magiran (http://www.magiran.com/), Iranian Research Institute for Information Science and Technology (IranDoc) (https://irandoc.ac.ir), Scientific Information Database (SID) (http://www.sid.ir/), Iranian National Library (http: //www.nlai.ir/) and Regional Information Center for Science and Technology (RICST) (http://en.ricest.ac.ir/) and seven English international databases including Scopus, PubMed/Medline, Cochrane Library, Science Direct, Web of Science, Embase, PsycINFO, as well as the Google Scholar search engine. For this purpose, the medical subject headings (MeSH) keywords for English databases and their Persian equivalents were used for Persian databases. These keywords include: 'Incidence', 'Prevalence', 'Age of Onset', 'Epidemiology', 'MS', 'Multiple Sclerosis', and 'Iran'. A combination of words was searched with the AND \& OR Boolean operators. The combined search was as follows: ("Multiple Sclerosis"[Title/Abstract] OR "Disseminated Sclerosis"[Title/Abstract] OR "MS"[Title/Abstract]) AND ("Age of Onset"[Title/Abstract] OR "Incidence"[All fields] OR "Prevalence"[Title/Abstract] OR "Epidemiology"[Title/Abstract]) AND ("Iran"[Title/Abstract/Affiliation]). The search period was determined to be until April 2017. We investigated the search strategy with a list of references for referenced articles and relevant overviews to identify potentially deleted articles. Finally, we contacted to corresponding author for more information through email.

\section{Inclusion \& exclusion criteria}

Inclusion criteria according to PICO (according to Evidence Based Medicine) [21]: population: epidemiological studies on MS patients; intervention: Poser or McDonald criteria to confirm MS; comparison: that can show the prevalence of MS clinical courses, positive MS family history and age of disease onset based on region; outcome: estimating the prevalence of MS clinical courses, positive MS family history, mean of age of disease onset, and disability in patients with MS.

The exclusion criteria were: nonrandom sample size; sample size other than MS patients; non-Iranian sample size; qualitative studies; non-relevance to the subject; review articles, case reports and letter to editor and duplicates.

\section{Quality assessment}

The researchers assessed the quality of selected articles using a scoring system (10 points) based on the modified Newcastle Ottawa Scale for cross-sectional studies [22]. The minimum score for entering the quantitative metaanalysis process was five and the articles that received the qualitative assessment score entered the process of data extraction and meta-analysis.

\section{Data extraction}

Data extraction was performed independently by two researchers, and in case of disagreement, with a third researcher. Data extraction sheet included first author, year of publication, year of study, study design, sample size, location of study, geographical area, province, prevalence and number of each MS clinical courses (RR, PP, SP and $\mathrm{RP})$, mean and standard deviation (SD) of the onset of the disease, mean and SD of the expanded disability status scale (EDSS), and the MS diagnostic method. 


\section{Statistical analysis}

In order to estimate the standard error and the prevalence in each study, normal distribution and binomial distribution were used, respectively. To evaluate the effect of gender (male to female ratio) on each of the clinical courses (RR, PP, SP and RP) of MS and positive family history of MS, odds ratio (OR) and for age at onset of disease and EDSS, standard mean difference (SMD) was used. Cochran's Q test and $\mathrm{I}^{2}$ index were used to study the heterogeneity of the studies. In moderate heterogeneity $\left(\mathrm{I}^{2}=25-49 \%\right)$, substantial heterogeneity $\left(\mathrm{I}^{2}=50-74 \%\right)$ and high heterogeneity $\left(\mathrm{I}^{2}=75-100 \%\right)$, a random effects model was used and in low heterogeneity $\left(\mathrm{I}^{2}<25 \%\right)$, a fixed effects model was adopted [23]. In this study, the sensitivity of the analysis were also examined. The subgroup analysis was performed in terms of geographic area. The publication bias was studied based on funnel plot as well as Egger and Begg's tests. Meta-regression model was used for each of the clinical courses of MS, and age of onset of the disease based on the year of study. Finally, the data were analyzed using comprehensive meta-analysis version 2. The significance level of the tests was considered less than 0.05 .

\section{Results}

\section{Study characteristics \& methodological quality}

Of the 460 primary searches using the search strategy, 230 duplicate studies and 140 irrelevant studies were deleted and 90 potentially relevant studies were retrieved. By reviewing the full text of the articles, 50 articles were excluded from the study (non-MS or non-Iranian patients $(n=23)$, nonrandom sample size $(n=17)$, review articles, case reports and letter to editor $(n=5)$ and inadequate data according to the data extraction checklists $(n=5))$. Finally, 40 studies with a sample of $42,472 \mathrm{MS}$ patients were reviewed. All studies had to score more than five in quality assessment. The flow diagram of the study and selection identifier is illustrated in Figure 1 and the characteristics of the studies are shown in Table 1.

\section{Clinical courses in Iranian MS patients Pooled prevalence}

The prevalence of clinical courses of RR, PP, SP and RP in patients with MS was 77.1\% (95\% CI: 72.2-81.3), 6.2\% (95\% CI: 4.1-9.2), 9.5\% (95\% CI: 6.8-13.1) and 0.4\% (95\% CI: 0.2-0.8), respectively (Figure 2).

\section{Meta-regression}

Meta-regression model for clinical courses of RR, PP, SP and RP based on the year of study is shown in Figure 3 and p-value for RR, PP, SP and RP is $0.53,0.91,0.23$ and 0.67 , respectively and were not statistically significant.

\section{Subgroup analysis}

The prevalence of MS clinical courses in the five geographical regions of Iran was shown in Table 2 and there was no significant difference in clinical courses of RR, PP, SP $(p>0.05)$ but in RP this difference was significant $(\mathrm{p}=0.015)($ Table 2$)$.

\section{Pooled prevalence based on gender}

The prevalence of clinical courses of MS (male vs. female) was estimated for RR (68.4 vs. $77.9 \%)$, PP (10.2 vs. $5.8 \%)$, SP (14.8 vs. $12.8 \%)$ and RP (0.6 vs. $0.4 \%)$ (Table 2$)$.

\section{Age at the onset of disease}

Pooled estimate

The mean age of disease onset in 17 studies with a sample size of 30,088 MS patients was 28.9 years (95\% CI: 27.8-30.1). The lowest and highest age of disease onset was studied in Mazandaran (25.3 years) and Qom (34.2 years), respectively (Figure 4A).

\section{Meta-regression}

Meta-regression for the mean age of disease onset based on the year of study is shown in Figure 5 and was not statistically significant $(\mathrm{p}=0.46)$. 


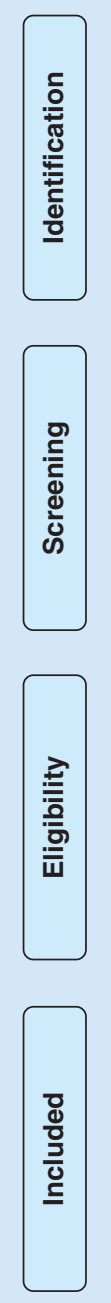

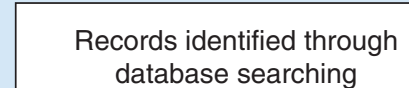
database searching $(n=440)$
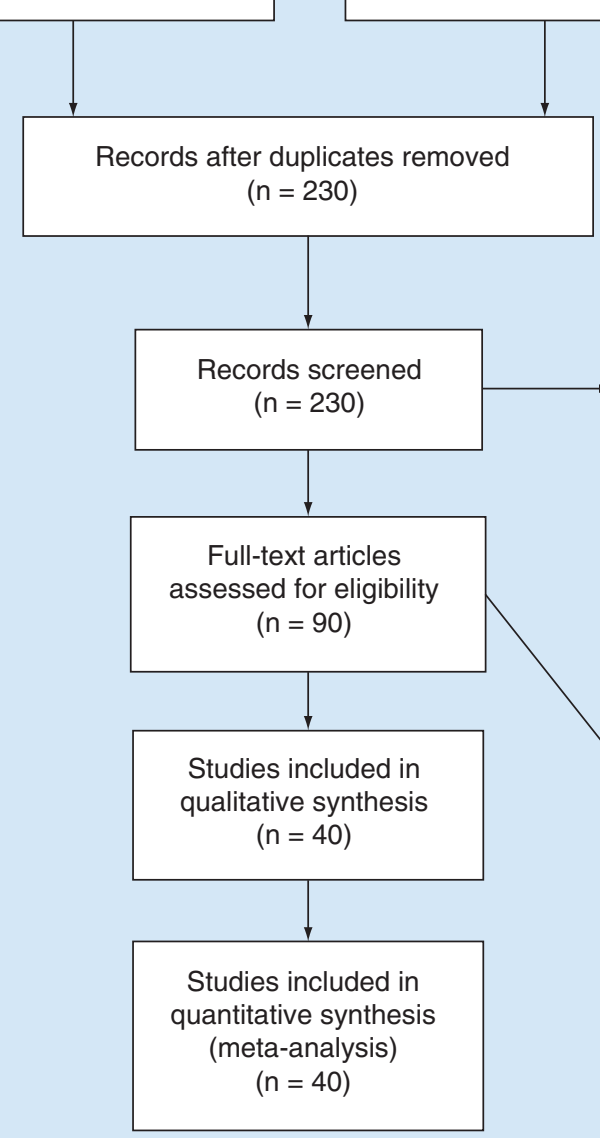

Records excluded due to irrelevancy $(n=140)$

Full-text articles excluded, $(n=50)$ With reasons: Non-MS or non-Iranian patients ( $n=23)$, non-randomized $(n=17)$, review articles, case reports and letter to editor $(n=5)$ and inadequate data according to the data extraction checklists $(n=5)$

\section{Figure 1. PRISMA flowchart.}

EDSS: Expanded Disability Status Scale; MS: Multiple sclerosis.

\section{Subgroup analysis}

Subgroup analysis of the mean age of disease onset in MS patients in terms of region in Table 2 shows that the lowest and highest mean age of disease onset was in the Eastern region (27.67 years) and Northern region (28.62 years), respectively. There was no significant difference $(\mathrm{p}=0.129)$ (Table 2$)$.

\section{Pooled estimate based on gender}

The mean age of disease onset in males and females patients with MS was 28.67 years (95\% CI: 27.7-29.6) and 27.96 (95\% CI: 26.7-29.2), respectively. The difference was statistically significant ( $<0.001)$ (Table 3).

\section{Disability based on EDSS \\ Pooled estimate}

In 15 studies with 5374 MS patients, the mean EDSS was 3.13 (95\% CI: 2.61-3.65) (Figure 4B).

\section{Subgroup analysis}

The mean EDSS in patients with MS in the Center, East, North and South regions of Iran was 3.45, 2.90, 2.77 and 2.93 , respectively. There was no significant difference $(\mathrm{p}=0.516)$. 
(A)

Statistics for each study

\begin{tabular}{|c|c|c|c|c|c|}
\hline Study name & $\begin{array}{c}\text { Event } \\
\text { rate }\end{array}$ & $\begin{array}{c}\text { Lower } \\
\text { limit }\end{array}$ & $\begin{array}{c}\text { Upper } \\
\text { limit }\end{array}$ & Z-value & p-value \\
\hline Sahraian MA (2010) & 0.849 & 0.841 & 0.857 & 55.802 & 0.000 \\
\hline Saadatnia M (2007) & 0.879 & 0.863 & 0.893 & 26.802 & 0.000 \\
\hline Abedini M (2008) & 0.710 & 0.672 & 0.745 & 9.802 & 0.000 \\
\hline Hashemilar M (2011) & 0.630 & 0.600 & 0.659 & 8.126 & 0.000 \\
\hline Moghtaderi A (2012) & 0.658 & 0.591 & 0.720 & 4.456 & 0.000 \\
\hline Kalanie H (2003) & 0.880 & 0.827 & 0.918 & 9.157 & 0.000 \\
\hline Ebrahimi HA (2013) & 0.700 & 0.666 & 0.731 & 10.718 & 0.000 \\
\hline Rezaali S (2013) & 0.801 & 0.767 & 0.831 & 13.527 & 0.000 \\
\hline Izadi S (2015) & 0.615 & 0.598 & 0.631 & 13.199 & 0.000 \\
\hline Ashtari F (2011) & 0.833 & 0.801 & 0.861 & 14.596 & 0.000 \\
\hline Asgari A (2015) & 0.643 & 0.554 & 0.723 & 3.114 & 0.002 \\
\hline Hojjati SM (2015) & 0.910 & 0.869 & 0.939 & 10.799 & 0.000 \\
\hline Rezapoor A (2017) & 0.836 & 0.773 & 0.884 & 7.886 & 0.000 \\
\hline Karampoor S (2016) & 0.767 & 0.734 & 0.797 & 13.326 & 0.000 \\
\hline Kargarfard M (2012) & 0.641 & 0.588 & 0.691 & 5.036 & 0.000 \\
\hline Dehghan-Nayeria N (2017) & 0.703 & 0.618 & 0.776 & 4.454 & 0.000 \\
\hline Saberi A (2012) & 0.775 & 0.713 & 0.827 & 7.466 & 0.000 \\
\hline Alehashemi A (2015) & 0.840 & 0.755 & 0.900 & 6.079 & 0.000 \\
\hline Nabavi SM (2006) & 0.724 & 0.659 & 0.781 & 6.142 & 0.000 \\
\hline Torabipour A (2014) & 0.918 & 0.883 & 0.943 & 12.075 & 0.000 \\
\hline Izadi S (2015) & 0.710 & 0.692 & 0.727 & 20.456 & 0.000 \\
\hline \multirow[t]{2}{*}{ Ashjazadeh N (2015) } & 0.620 & 0.521 & 0.710 & 2.376 & 0.017 \\
\hline & 0.771 & 0.722 & 0.813 & 9.196 & 0.000 \\
\hline
\end{tabular}

Heterogeneity: $I^{2}: 98.19 ; p<0.001$. Random effects model

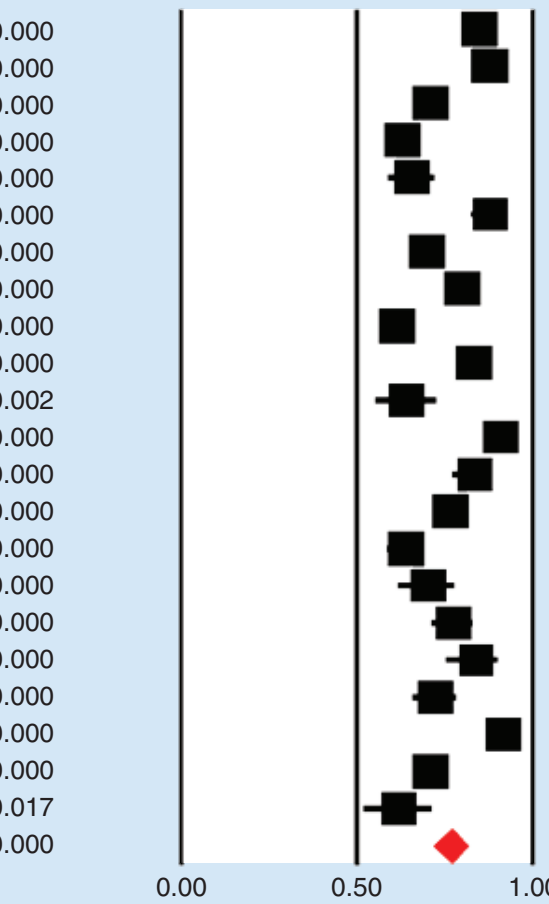

Relative weight

\subsection{1}

4.75

4.72

4.77

4.55

4.27

4.74

4.69

4.81

4.67

4.39

4.28

4.32

4.72

4.66

4.37

4.49

4.00

4.52

4.34

4.80

4.32

\section{Meta analysis}

(B)

\begin{tabular}{|c|c|c|c|c|c|c|c|}
\hline Study name & $\begin{array}{l}\text { Event } \\
\text { rate }\end{array}$ & $\begin{array}{c}\text { Lower } \\
\text { limit }\end{array}$ & $\begin{array}{l}\text { Upper } \\
\text { limit }\end{array}$ & Z-value & p-value & Event rate and $95 \% \mathrm{Cl}$ & \multirow{2}{*}{$\begin{array}{r}\begin{array}{c}\text { Relative } \\
\text { weight }\end{array} \\
5.73\end{array}$} \\
\hline Sahraian MA (2010) & 0.151 & 0.143 & 0.159 & -55.802 & 0.000 & & \\
\hline Saadatnia M (2007) & 0.017 & 0.012 & 0.024 & -21.647 & 0.000 & & 5.50 \\
\hline Abedini M (2008) & 0.058 & 0.042 & 0.080 & -15.719 & 0.000 & & 5.52 \\
\hline Hashemilar M (2011) & 0.026 & 0.018 & 0.038 & -18.234 & 0.000 & & 5.47 \\
\hline Moghtaderi A (2012) & 0.200 & 0.151 & 0.260 & -7.959 & 0.000 & & 5.53 \\
\hline Kalanie H (2003) & 0.070 & 0.042 & 0.115 & -9.334 & 0.000 & & 5.24 \\
\hline Ebrahimi HA (2013) & 0.280 & 0.249 & 0.313 & -11.706 & 0.000 & & 5.69 \\
\hline Rezaali S (2013) & 0.054 & 0.038 & 0.075 & -15.746 & 0.000 & & 5.51 \\
\hline Asgari A (2015) & 0.041 & 0.017 & 0.095 & -6.902 & 0.000 & & 4.57 \\
\hline Hojjati SM (2015) & 0.090 & 0.061 & 0.131 & -10.799 & 0.000 & & 5.43 \\
\hline Rezapoor A (2017) & 0.035 & 0.016 & 0.076 & -7.975 & 0.000 & & 4.74 \\
\hline Karampoor S (2016) & 0.094 & 0.074 & 0.118 & -17.494 & 0.000 & & 5.62 \\
\hline Kargarfard M (2012) & 0.103 & 0.074 & 0.141 & -11.914 & 0.000 & & 5.51 \\
\hline Dehghan-Nayeria N (2017) & 0.072 & 0.038 & 0.132 & -7.476 & 0.000 & & 5.02 \\
\hline Saberi A (2012) & 0.081 & 0.051 & 0.127 & -9.580 & 0.000 & & 5.32 \\
\hline Alehashemi A (2015) & 0.030 & 0.010 & 0.089 & -5.930 & 0.000 & & 4.04 \\
\hline Torabipour A (2014) & 0.034 & 0.019 & 0.060 & -11.052 & 0.000 & & 5.16 \\
\hline Izadi S (2015) & 0.008 & 0.005 & 0.012 & -21.620 & 0.000 & & \multirow{3}{*}{$\begin{array}{l}5.41 \\
4.99\end{array}$} \\
\hline \multirow[t]{2}{*}{ Ashjazadeh N (2015) } & 0.090 & 0.047 & 0.164 & -6.621 & 0.000 & & \\
\hline & 0.062 & 0.041 & 0.092 & -12.537 & 0.000 & & \\
\hline \multicolumn{6}{|c|}{ Heterogeneity: $I^{2}: 97.39 ; p<0.001$. Random effects model } & 0.50 & \\
\hline
\end{tabular}

\section{Meta analysis}

Figure 2. Prevalence of clinical courses of relapsing-remitting (A) primary progressive (B) secondary progressive (C) relapsing progressive (D) in Iranian multiple sclerosis patients. Random effects model. 
(C)

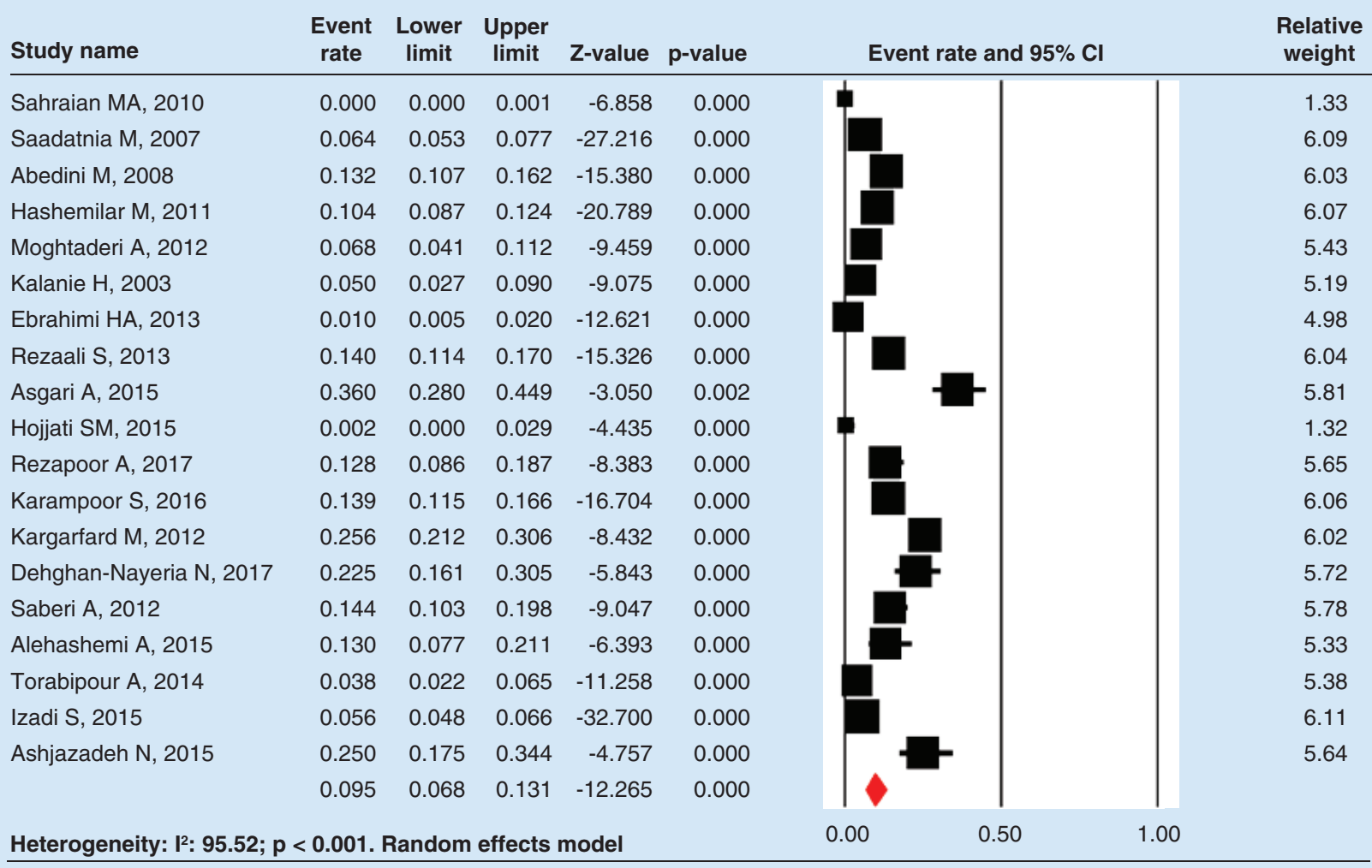

\section{Statistics for each study}

Meta analysis

(D)

Statistics for each study

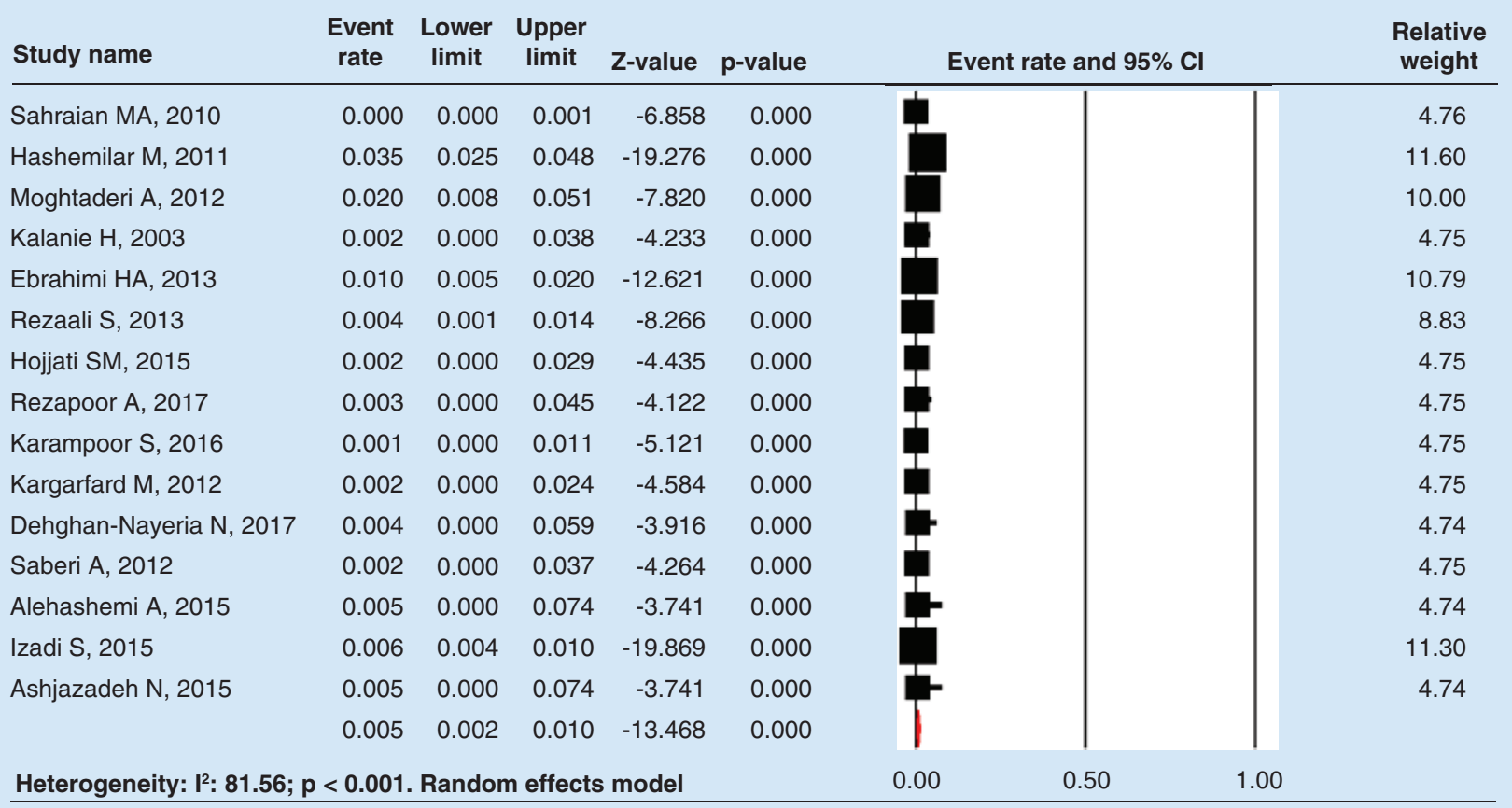

Meta analysis

Figure 2. Prevalence of clinical courses of relapsing-remitting (A) primary progressive (B) secondary progressive (C) relapsing progressive (D) in Iranian multiple sclerosis patients. Random effects model (cont.). 
Table 1. Summary of demographic characteristics in studies into a meta-analysis.

\begin{tabular}{|c|c|c|c|c|c|c|c|c|}
\hline \multirow[t]{2}{*}{ Study } & \multirow[t]{2}{*}{ Year of study } & \multirow[t]{2}{*}{ Study type } & \multirow[t]{2}{*}{ Place } & \multirow[t]{2}{*}{ Diagnostic criteria } & \multicolumn{3}{|c|}{ Sample size } & \multirow[t]{2}{*}{ Ref. } \\
\hline & & & & & All & Male & Female & \\
\hline Izadi S (2015) & 2013 & Population based & Fars & McDonald & 3354 & 665 & 2689 & [15] \\
\hline Ashjazadeh N (2015) & 2010 & Cross-sectional & Fars & McDonald & 100 & & & [16] \\
\hline Izadi S (2015) & 2011 & Population based & Fars & McDonald & 2535 & & & [17] \\
\hline Jajvandian R (2011) & 2005-2011 & Population based & North Khorasan & McDonald & 105 & & & [18] \\
\hline Majdinasab N (2012) & $2005-2011$ & Population based & Khuzestan & McDonald & 1057 & & & [24] \\
\hline Etemadifar M (2010) & $2003-2010$ & Cross-sectional & Isfahan & McDonald & 3522 & 806 & 2716 & [25] \\
\hline Dehghan-Nayeria N (2017) & 2016 & Cross-sectional & Tehran & McDonald & 128 & & & [26] \\
\hline Choobforouszade A (2016) & 2014 & Cross-sectional & Yazd & McDonald & 108 & & & [27] \\
\hline Etemadifar M (2013) & 2003-2013 & Population based & Isfahan & McDonald & 4536 & 1028 & 3508 & [8] \\
\hline Saman-Nezhad B (2012) & 2012 & Population based & Kermanshah & McDonald & 448 & 115 & 333 & [28] \\
\hline Moghtaderi A (2012) & 1996-2006 & Cross-sectional & $\begin{array}{l}\text { Sistan and } \\
\text { Balouchestan }\end{array}$ & McDonald & 206 & 65 & 141 & [29] \\
\hline Taraghi Z (2007) & 2006 & Cross-sectional & Mazandaran & McDonald & 101 & & & [30] \\
\hline Pourmemari MH (2011) & 2001-2005 & Cross-sectional & Zanjan & McDonald & 96 & & & [31] \\
\hline Lasemi E (2011) & 2010 & Cross-sectional & Tehran & McDonald & 400 & & & [32] \\
\hline Khoshravesh S (2016) & 2014 & Cross-sectional & Kordestan & McDonald & 800 & & & [33] \\
\hline Danesh-Sani SA (2013) & 2010-2011 & Cross-sectional & Razavi Khorasan & McDonald & 510 & & & [34] \\
\hline Ashtari F (2011) & 2007 & Cross-sectional & Isfahan & McDonald & 593 & 100 & 493 & [35] \\
\hline Hojjati SM (2015) & $2002-2012$ & Cross-sectional & Mazandaran & McDonald & 266 & 78 & 188 & [36] \\
\hline Saadat SMS (2014) & 2010 & Cross-sectional & Guilan & McDonald & 160 & & & [37] \\
\hline Rezapoor A (2017) & 2013 & Cross-sectional & Fars & McDonald & 171 & & & [38] \\
\hline Torabipour A (2014) & 2012 & Cross-sectional & Khuzestan & McDonald & 332 & & & [39] \\
\hline Alehashemi A (2015) & 2013-2014 & Cross-sectional & Razavi Khorasan & McDonald & 100 & 25 & 75 & [40] \\
\hline Taraghi Z (2010) & 2005-2006 & Cross-sectional & Mazandaran & McDonald & 100 & & & [41] \\
\hline Karampoor S (2016) & 2010 & Cross-sectional & Tehran & McDonald & 700 & & & [42] \\
\hline Ayatollahi P (2007) & 2007 & Cross-sectional & Tehran & McDonald & 106 & & & [43] \\
\hline Ghajarzadeh M (2012) & 2008-2009 & Cross-sectional & Tehran & McDonald & 100 & & & [44] \\
\hline Nabavi SM (2006) & 2002 & Cross-sectional & Tehran & McDonald & 203 & & & [45] \\
\hline Mohammadi K (2013) & 2009-2010 & Cross-sectional & Tehran & McDonald & & & 226 & [46] \\
\hline Saberi A (2012) & 2008-2010 & Cross-sectional & Guilan & McDonald & 209 & 54 & 155 & [47] \\
\hline Sahraian MA (2010) & 1999-2010 & Cross-sectional & Tehran & McDonald & 8146 & 2258 & 5888 & [48] \\
\hline Saadatnia M (2007) & 2003-2006 & Cross-sectional & Isfahan & McDonald & 1718 & 388 & 1083 & [49] \\
\hline Khanjani Z (2012) & 2011 & Cross-sectional & Tehran & McDonald & 80 & 24 & 54 & [50] \\
\hline Kargarfard M (2012) & 2010 & Cross-sectional & Isfahan & McDonald & 328 & 208 & 120 & [51] \\
\hline Abedini M (2008) & 2007 & Cross-sectional & Mazandaran & McDonald & 582 & 161 & 421 & [52] \\
\hline Kalanie H (2003) & 1996-2001 & Cross-sectional & Tehran & McDonald & 200 & 56 & 144 & [53] \\
\hline Elhami SR (2011) & 1989-2009 & Population based & Tehran & McDonald & 7896 & 2008 & 5588 & [54] \\
\hline Hashemilar M (2011) & 2005-2009 & Population based & East Azerbaijan & McDonald & 1000 & 269 & 731 & [55] \\
\hline Ebrahimi HA (2013) & 2013 & Population based & Kerman & McDonald & 762 & 188 & 744 & [56] \\
\hline Asgari A (2015) & 2009-2014 & Cross-sectional & Tehran & McDonald & 122 & 45 & 77 & [57] \\
\hline Rezaali S (2013) & 2011 & Population based & Qom & McDonald & 592 & 132 & 449 & [58] \\
\hline
\end{tabular}

\section{EDSS based on gender}

The mean EDSS in males and females patients with MS was 3.48 (95\% CI: 2.76-4.20) and 3.14 (95\% CI:

2.46-3.83), respectively. There was no significant difference $(\mathrm{p}=0.907)$ (Table 3 ). 

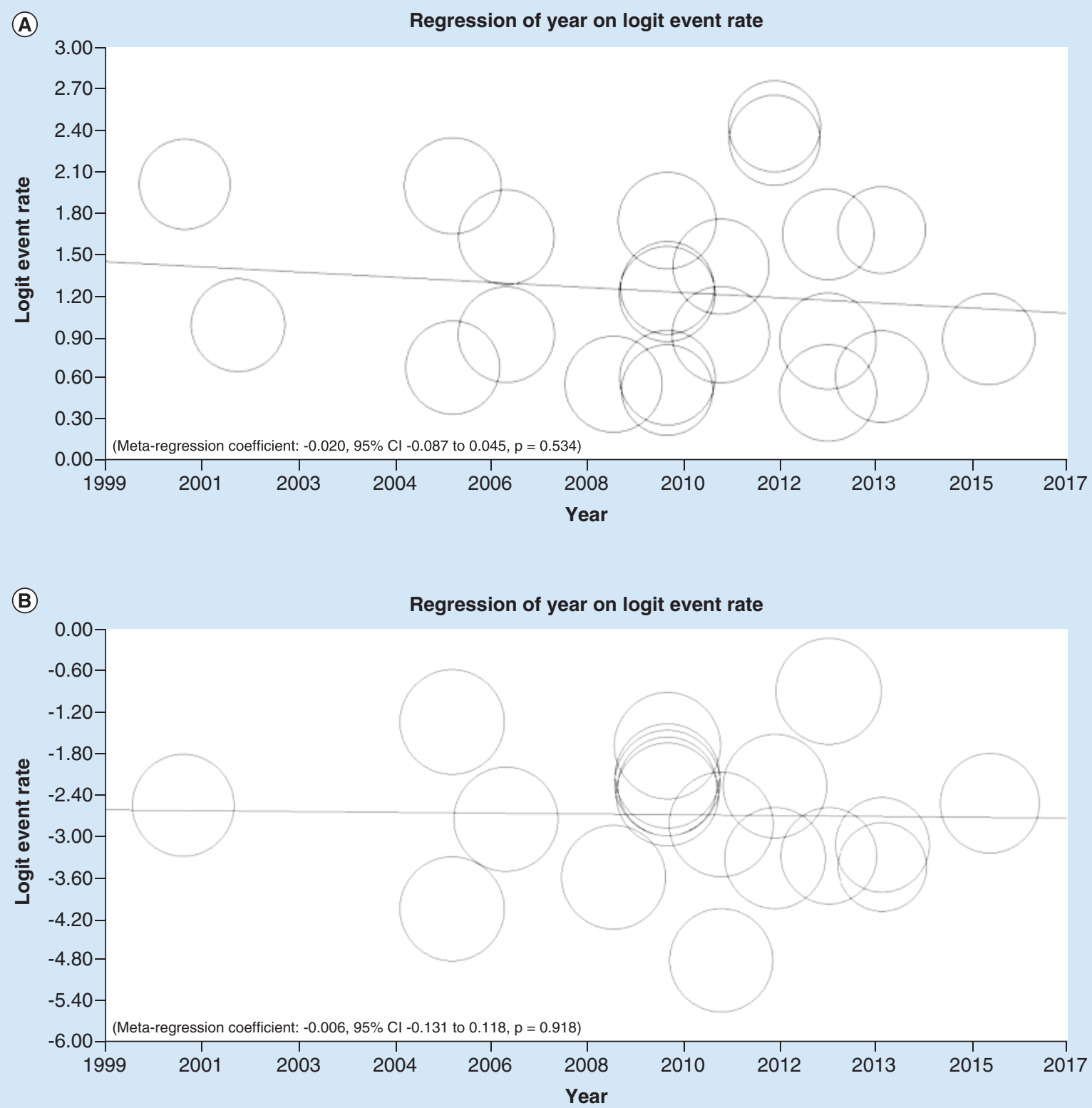

Figure 3. Association of clinical courses of relapsing-remitting (A) primary progressive (B) secondary progressive (C) relapsing progressive (D) and year of study in Iranian multiple sclerosis patients.

\section{Family history of MS}

Pooled prevalence

The prevalence of positive family history in patients with MS was 8.9\% (95\% CI: 6.9-11.4). The lowest prevalence was in a study in Kermanshah (1.2\%) and the highest prevalence was in a study in Isfahan (20.1\%) (Figure 6).

\section{Subgroup analysis}

The family history of MS was estimated in the Central 11\%, Eastern $10.1 \%$, Northern $7.1 \%$ and Western $3.8 \%$ regions of Iran. The difference in family history of MS between regions was not significant $(\mathrm{p}=0.101)$ (Table 2$)$. 

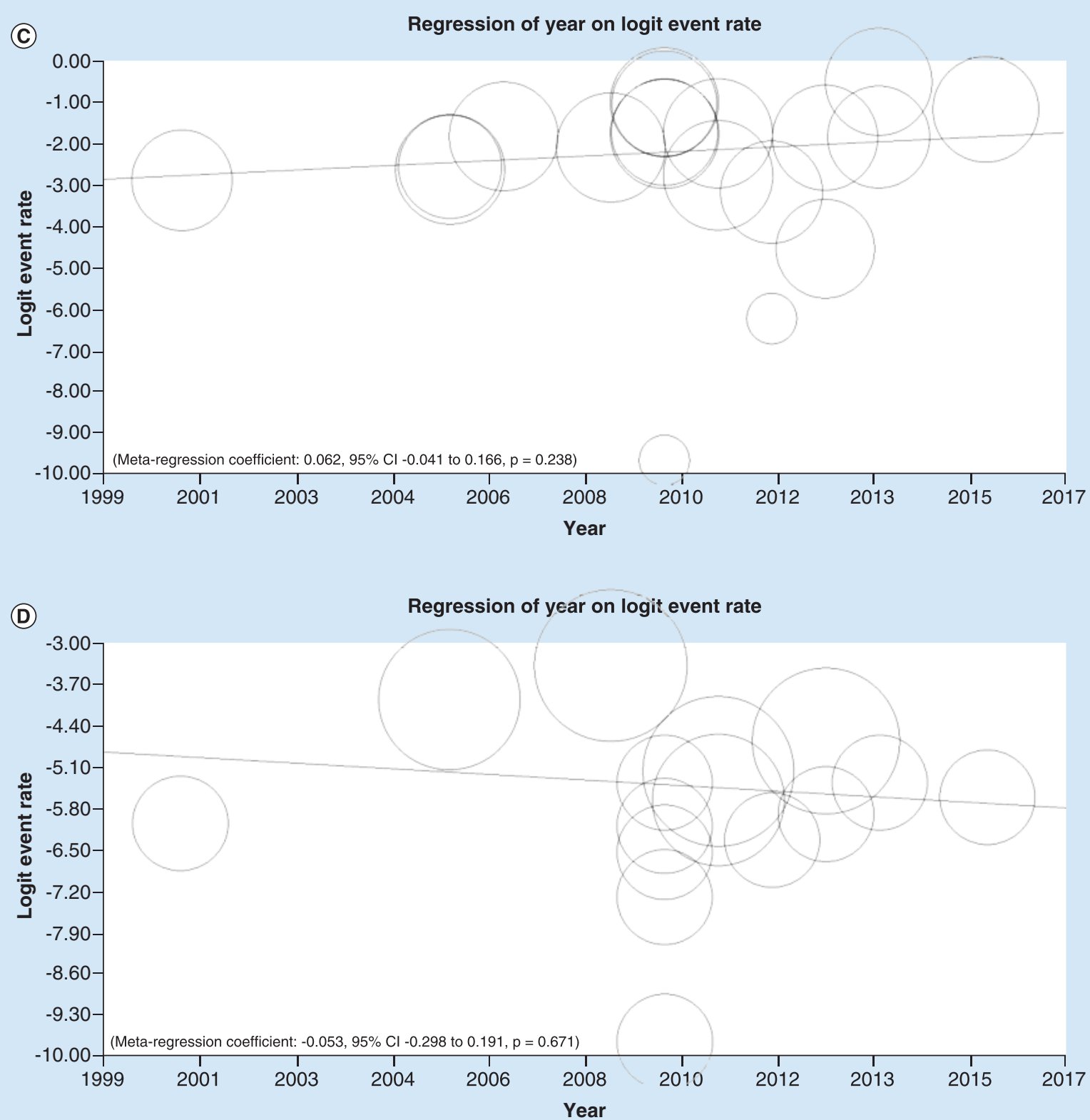

Figure 3. Association of clinical courses of relapsing-remitting (A) primary progressive (B) secondary progressive (C) relapsing progressive (D) and year of study in Iranian multiple sclerosis patients (cont.).

\section{Pooled prevalence based on gender}

In Table 3, the prevalence of MS family history was shown in males (13\%; 95\% CI: 10.6-15.8) and females (8.6\%; 95\% CI: 4.5-15.9). The positive family history of MS in men were significantly higher than women $(\mathrm{OR}=1.37$; $\mathrm{p}=0.018)$.

\section{Sensitivity analysis}

Sensitivity analysis showed a robust point estimate by removing a single study at the same time for the clinical courses (RR, PP, SP and RP), the mean age of disease onset, EDSS and MS family history (Supplementary Figure $1)$. 
(A)

\begin{tabular}{|c|c|c|c|c|c|c|c|}
\hline Study name & Mean & $\begin{array}{l}\text { Standard } \\
\text { error }\end{array}$ & Variance & $\begin{array}{l}\text { Lower } \\
\text { limit }\end{array}$ & $\begin{array}{l}\text { Upper } \\
\text { limit }\end{array}$ & Z-value & p-value \\
\hline Sahraian MA (2010) & 27.240 & 0.092 & 0.008 & 27.059 & 27.421 & 295.499 & 0.000 \\
\hline Elhami SR (2011) & 27.530 & 0.091 & 0.008 & 27.351 & 27.709 & 301.269 & 0.000 \\
\hline Saadatnia M (2007) & 25.360 & 0.207 & 0.043 & 24.953 & 25.767 & 122.226 & 0.000 \\
\hline Etemadifar M (2010) & 28.200 & 0.152 & 0.023 & 27.903 & 28.497 & 185.952 & 0.000 \\
\hline Abedini M (2008) & 26.900 & 0.344 & 0.118 & 26.226 & 27.574 & 78.187 & 0.000 \\
\hline Hashemilar M (2011) & 32.500 & 0.262 & 0.069 & 31.986 & 33.014 & 123.824 & 0.000 \\
\hline Etemadifar M (2013) & 33.100 & 0.141 & 0.020 & 32.824 & 33.376 & 234.661 & 0.000 \\
\hline Kalanie H (2003) & 27.000 & 0.523 & 0.274 & 25.974 & 28.026 & 51.600 & 0.000 \\
\hline Majdinasab N (2012) & 27.600 & 0.252 & 0.064 & 27.106 & 28.094 & 109.429 & 0.000 \\
\hline Rezaali S (2013) & 34.250 & 0.370 & 0.137 & 33.524 & 34.976 & 92.490 & 0.000 \\
\hline Asgari A (2015) & 29.900 & 0.561 & 0.315 & 28.800 & 31.000 & 53.267 & 0.000 \\
\hline Rezapoor A (2017) & 28.100 & 0.581 & 0.338 & 26.961 & 29.239 & 48.349 & 0.000 \\
\hline Taraghi Z (2007) & 27.200 & 0.799 & 0.638 & 25.634 & 28.766 & 34.042 & 0.000 \\
\hline Kargarfard M (2012) & 26.940 & 0.250 & 0.063 & 26.450 & 27.430 & 107.705 & 0.000 \\
\hline Dehghan-Nayeria N (2017) & 30.100 & 0.645 & 0.416 & 28.835 & 31.365 & 46.650 & 0.000 \\
\hline Choobforouszade A (2016) & 32.400 & 0.693 & 0.480 & 31.042 & 33.758 & 46.765 & 0.000 \\
\hline \multirow[t]{2}{*}{ Saberi A (2012) } & 27.800 & 0.574 & 0.330 & 26.675 & 28.925 & 48.422 & 0.000 \\
\hline & 28.943 & 0.608 & 0.369 & 27.752 & 30.134 & 47.625 & 0.000 \\
\hline
\end{tabular}

Statistics for each study
Mean and $95 \% \mathrm{Cl}$ weight

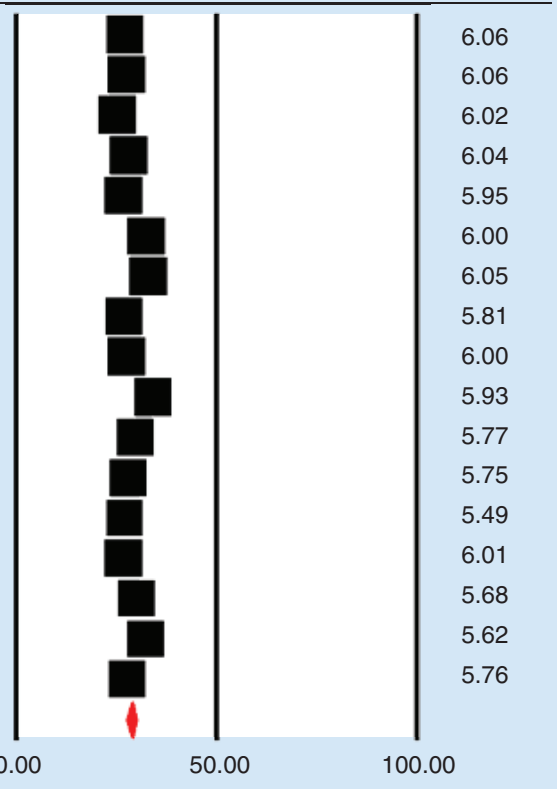

Meta analysis

(B)

\begin{tabular}{|c|c|c|c|c|c|c|c|c|c|}
\hline Study name & Mean & $\begin{array}{l}\text { Standard } \\
\text { error }\end{array}$ & Variance & $\begin{array}{l}\text { Lower } \\
\text { limit }\end{array}$ & $\begin{array}{l}\text { Upper } \\
\text { limit }\end{array}$ & Z-value & p-value & Mean and $95 \% \mathrm{Cl}$ & $\begin{array}{c}\text { Relative } \\
\text { weight }\end{array}$ \\
\hline Saadatnia M (2007) & 2.700 & 0.031 & 0.001 & 2.639 & 2.761 & 86.086 & 0.000 & & 6.86 \\
\hline Kalanie H (2003) & 2.100 & 0.099 & 0.010 & 1.906 & 2.294 & 21.213 & 0.000 & & 6.80 \\
\hline Ebrahimi HA (2013) & 4.500 & 0.069 & 0.005 & 4.365 & 4.635 & 65.379 & 0.000 & & 6.84 \\
\hline Asgari A (2015) & 3.200 & 0.212 & 0.045 & 2.785 & 3.615 & 15.105 & 0.000 & & 6.58 \\
\hline Hojjati SM (2015) & 1.670 & 0.047 & 0.002 & 1.577 & 1.763 & 35.373 & 0.000 & & 6.85 \\
\hline Saadat SMS (2014) & 1.970 & 0.083 & 0.007 & 1.807 & 2.133 & 23.732 & 0.000 & & 6.82 \\
\hline Taraghi Z (2010) & 2.960 & 0.177 & 0.031 & 2.613 & 3.307 & 16.723 & 0.000 & & 6.66 \\
\hline Ghajarzadeh M (2012) & 4.800 & 0.520 & 0.270 & 3.781 & 5.819 & 9.231 & 0.000 & & 5.44 \\
\hline Ayatollahi P (2007) & 4.400 & 0.175 & 0.031 & 4.057 & 4.743 & 25.167 & 0.000 & & 6.67 \\
\hline Rezapoor A (2017) & 2.100 & 0.168 & 0.028 & 1.770 & 2.430 & 12.482 & 0.000 & & 6.68 \\
\hline Karampoor S (2016) & 4.000 & 0.057 & 0.003 & 3.889 & 4.111 & 70.553 & 0.000 & & 6.85 \\
\hline Kargarfard M (2012) & 3.390 & 0.099 & 0.010 & 3.196 & 3.584 & 34.299 & 0.000 & & 6.80 \\
\hline Saberi A (2012) & 4.500 & 0.111 & 0.012 & 4.283 & 4.717 & 40.660 & 0.000 & & 6.79 \\
\hline Alehashemi A (2015) & 2.900 & 0.230 & 0.053 & 2.449 & 3.351 & 12.609 & 0.000 & & 6.53 \\
\hline \multirow[t]{2}{*}{ Torabipour A (2014) } & 2.200 & 0.088 & 0.008 & 2.028 & 2.372 & 25.054 & 0.000 & & 6.82 \\
\hline & 3.137 & 0.265 & 0.070 & 2.616 & 3.657 & 11.817 & 0.000 & & \\
\hline \multicolumn{8}{|c|}{ Heterogeneity: $I^{2}: 99.37 ; p<0.001$. Random effects model } & 4.00 & \\
\hline
\end{tabular}

Meta analysis

Figure 4. The mean age of disease onset (A) and the mean EDSS (B) in Iranian multiple sclerosis patients.

EDSS: Expanded disability status scale. 
Table 2. Subgroup analysis of of relapsing remitting, primary progressive, secondary progressive and relapsing progressive, age of disease onset, family history and ESDD in multiple sclerosis patients based on region.

\begin{tabular}{|c|c|c|c|c|c|c|c|}
\hline \multirow[t]{2}{*}{ Variables } & & \multirow[t]{2}{*}{ Studies $\left(\mathbf{N}^{\dagger}\right)$} & \multirow[t]{2}{*}{ Sample (N) } & \multicolumn{2}{|c|}{ Heterogeneity } & \multirow[t]{2}{*}{$95 \% \mathrm{Cl}^{\ddagger}$} & \multirow[t]{2}{*}{ Overall (\%) } \\
\hline & & & & 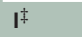 & p-value & & \\
\hline \multirow{5}{*}{$\begin{array}{l}\text { Relapsing } \\
\text { remitting }\end{array}$} & Center & 10 & 12,730 & 95.71 & $<0.001$ & $73.4-83$ & 78.5 \\
\hline & East & 2 & 306 & 90.47 & 0.001 & $53.7-89.2$ & 75.6 \\
\hline & North & 5 & 2190 & 0 & 0.42 & $0.42-1.12$ & 0.69 \\
\hline & South & 6 & 7204 & 96.89 & $<0.001$ & $67.7-80.7$ & 74.7 \\
\hline & \multicolumn{7}{|c|}{ Test for subgroup differences: $Q=0.895, \mathrm{df}(\mathrm{Q})=3, \mathrm{p}=0.827$} \\
\hline \multirow{5}{*}{$\begin{array}{l}\text { Primary } \\
\text { progressive }\end{array}$} & Center & 8 & 11,934 & 96.80 & $<0.001$ & $3.7-11.2$ & 6.6 \\
\hline & East & 2 & 306 & 91.34 & 0.001 & $1.2-42.2$ & 8.7 \\
\hline & North & 4 & 2057 & 87.65 & $<0.001$ & $3.3-9.8$ & 5.8 \\
\hline & South & 5 & 3900 & 98.78 & $<0.001$ & $0.5-24.6$ & 5 \\
\hline & \multicolumn{7}{|c|}{ Test for subgroup differences: $Q=0.291, d f(Q)=3, p=0.962$} \\
\hline \multirow{5}{*}{$\begin{array}{l}\text { Secondary } \\
\text { progressive }\end{array}$} & Center & 8 & 11,934 & 96.60 & $<0.001$ & $6.9-19.7$ & 11.9 \\
\hline & East & 2 & 306 & 67.89 & 0.078 & $4.9-17.3$ & 9.4 \\
\hline & North & 4 & 2057 & 77.62 & 0.004 & $8.3-16.1$ & 11.6 \\
\hline & South & 5 & 3900 & 95.65 & $<0.001$ & $2.7-13.9$ & 6.3 \\
\hline & \multicolumn{7}{|c|}{ Test for subgroup differences: $Q=2.213, d f(Q)=3, p=0.529$} \\
\hline \multirow{5}{*}{$\begin{array}{l}\text { Relapsing } \\
\text { progressive }\end{array}$} & Center & 6 & 10,094 & 34.55 & 0.177 & $0-0.5$ & 0.1 \\
\hline & East & 2 & 306 & 0 & 0.348 & $0.7-4.2$ & 1.7 \\
\hline & North & 3 & 1475 & 74.48 & 0.020 & $0.1-7$ & 0.8 \\
\hline & South & 4 & 3568 & 0 & 0.618 & $0.5-1$ & 0.7 \\
\hline & \multicolumn{7}{|c|}{ Test for subgroup differences: $Q=10.475, \mathrm{df}(\mathrm{Q})=3, \mathrm{p}=0.015$} \\
\hline \multirow{7}{*}{$\begin{array}{l}\text { Positive family } \\
\text { history }\end{array}$} & Center & 6 & 3599 & 88.63 & $<0.001$ & $8.1-14.9$ & 11 \\
\hline & East & 2 & 716 & 89.90 & 0.002 & $4.1-22.8$ & 10.1 \\
\hline & North & 3 & 1201 & 0.87 & 0.99 & $5.8-8.7$ & 7.1 \\
\hline & West & 2 & 1248 & 96.08 & $<0.001$ & $0.4-26.6$ & 3.8 \\
\hline & \multicolumn{7}{|c|}{ Test for subgroup differences: $Q=6.23, d f(Q)=3, p=0.101$} \\
\hline & & \multirow[t]{2}{*}{ Studies (N) } & \multirow[t]{2}{*}{ Sample (N) } & \multicolumn{2}{|c|}{ Heterogeneity } & \multirow[t]{2}{*}{$95 \% \mathrm{Cl}$} & \multirow[t]{2}{*}{ Overall (mean) } \\
\hline & & & & $1^{2}$ & p-value & & \\
\hline \multirow{4}{*}{$\begin{array}{l}\text { Age at onset of } \\
\text { disease }\end{array}$} & Center & 11 & 26,968 & 99.47 & $<0.001$ & $27.75-30.74$ & 29.25 \\
\hline & North & 4 & 1892 & 98.49 & $<0.001$ & $25.24-31.99$ & 28.62 \\
\hline & South & 2 & 1228 & 0 & 0.430 & $27.22-28.13$ & 27.67 \\
\hline & \multicolumn{7}{|c|}{ Test for subgroup differences: $Q=4.08, d f(Q)=2, p=0.129$} \\
\hline \multirow[t]{5}{*}{ EDSS } & Center & 7 & 3274 & 98.96 & $<0.001$ & $2.83-4.07$ & 3.45 \\
\hline & East & 1 & 100 & 0 & - & $2.44-3.35$ & 2.90 \\
\hline & North & 4 & 735 & 99.48 & $<0.001$ & $1.56-3.98$ & 2.77 \\
\hline & South & 3 & 1265 & 99.59 & $<0.001$ & $1.18-4.68$ & 2.93 \\
\hline & \multicolumn{7}{|c|}{ Test for subgroup differences: $Q=2.283, d f(Q)=3, p=0.516$} \\
\hline
\end{tabular}

Publication bias

Publication bias was not significant for clinical courses of RR (p-value for Egger and Begg's tests was 0.824 and 0.127, respectively), SP (p-value for Egger and Begg's tests was 0.686 and 0.207, respectively), the mean age of disease onset (p-value for Egger and Begg's tests was 0.456 and 0.108, respectively), EDSS (p-value for Egger and 


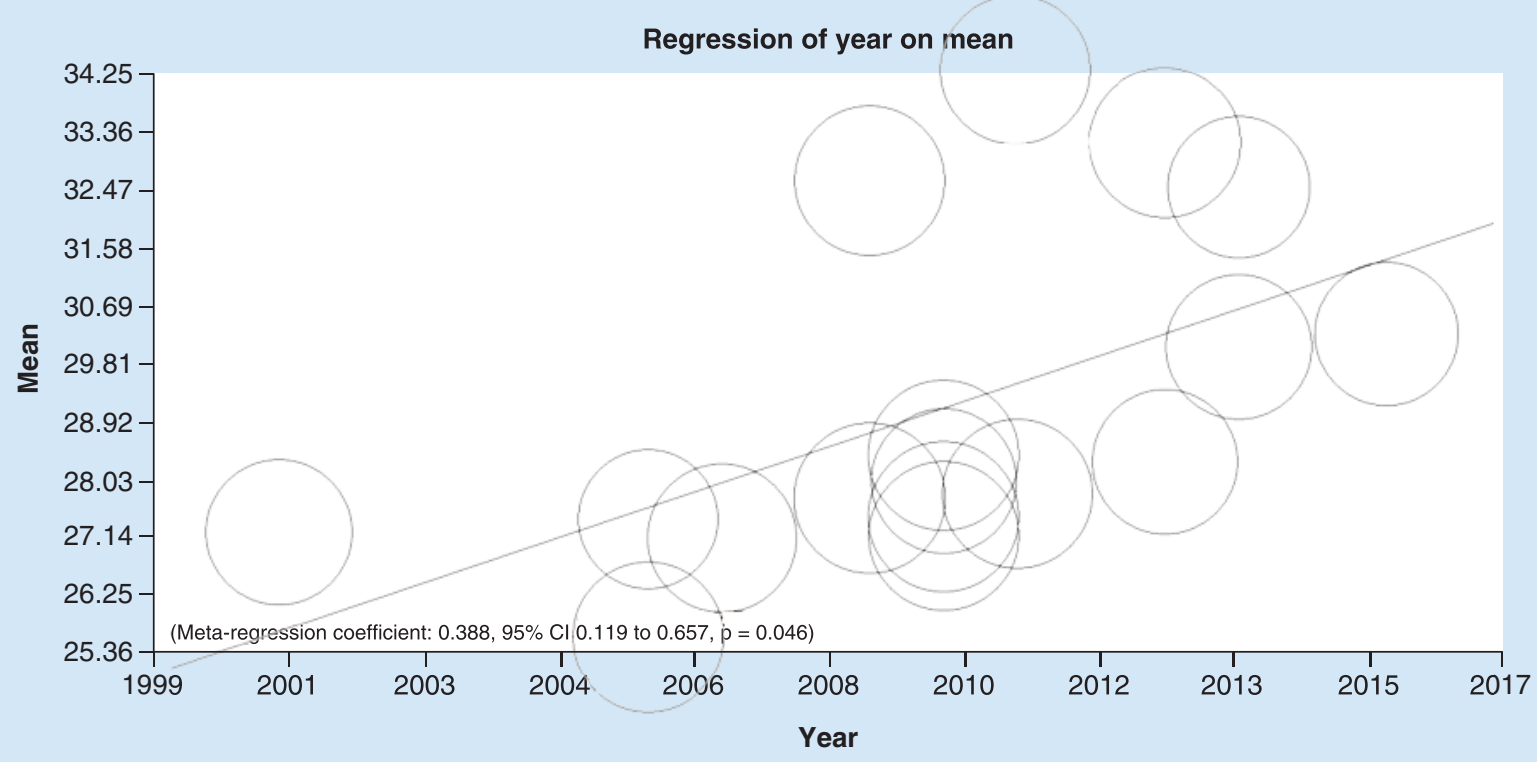

Figure 5. Association of the mean age of disease onset and year of study in Iranian multiple sclerosis.

\begin{tabular}{|c|c|c|c|c|c|c|c|c|c|}
\hline \multirow[t]{2}{*}{ Variables } & & \multirow[t]{2}{*}{ Studies $\left(\mathbf{N}^{\dagger}\right)$} & \multirow[t]{2}{*}{ Sample (N) } & \multicolumn{2}{|c|}{ Heterogeneity } & \multirow[t]{2}{*}{$95 \% \mathrm{Cl}^{\ddagger}$} & \multirow[t]{2}{*}{ Pooled (\%) } & \multicolumn{2}{|c|}{ Ratio of males to females } \\
\hline & & & & $\mathrm{I}^{\ddagger}$ & p-value & & & $\mathrm{OR}^{\S}(95 \% \mathrm{Cl})$ & p-value \\
\hline \multirow[t]{2}{*}{$\begin{array}{l}\text { Relapsing } \\
\text { remitting }\end{array}$} & Male & 8 & 3515 & 93.09 & $<0.001$ & $60.1-75.6$ & 68.4 & $\begin{array}{l}0.88 \text { (95\% Cl: } \\
0.84-0.92)\end{array}$ & $<0.001$ \\
\hline & Female & 9 & 9150 & 97.32 & $<0.001$ & $69.9-84.3$ & 77.9 & & \\
\hline \multirow[t]{2}{*}{$\begin{array}{l}\text { Primary } \\
\text { progressive }\end{array}$} & Male & 8 & 3515 & 92.75 & $<0.001$ & $6.2-16.3$ & 10.2 & $\begin{array}{l}1.71(95 \% \mathrm{Cl}: \\
1.55-1.90)\end{array}$ & $<0.001$ \\
\hline & Female & 9 & 9150 & 94.19 & $<0.001$ & $3.6-9$ & 5.8 & & \\
\hline \multirow[t]{2}{*}{$\begin{array}{l}\text { Secondary } \\
\text { progressive }\end{array}$} & Male & 8 & 3515 & 93.27 & $<0.001$ & $8.4-24.9$ & 14.8 & $\begin{array}{l}1.55(95 \% \mathrm{Cl}: \\
1.14-2.09)\end{array}$ & 0.004 \\
\hline & Female & 9 & 9150 & 93.9 & $<0.001$ & $8.3-19.1$ & 12.8 & & \\
\hline \multirow[t]{2}{*}{$\begin{array}{l}\text { Primary } \\
\text { progressive }\end{array}$} & Male & 4 & 2713 & 86.30 & $<0.001$ & $0-0.87$ & 0.6 & $\begin{array}{l}2.49(95 \% \mathrm{Cl}: \\
1.33-4.66)\end{array}$ & 0.004 \\
\hline & Female & 5 & 7343 & 82.91 & $<0.001$ & $0.01-2.1$ & 0.4 & & \\
\hline \multirow[t]{2}{*}{$\begin{array}{l}\text { Positive family } \\
\text { history of MS }\end{array}$} & Male & 2 & 657 & 64.81 & 0.092 & $10.6-15.8$ & 13 & $\begin{array}{l}1.37(95 \% \mathrm{Cl}: \\
1.05-1.77)\end{array}$ & 0.018 \\
\hline & Female & 2 & 1814 & 93.54 & $<0.001$ & $4.5-15.9$ & 8.6 & & \\
\hline \multirow[t]{2}{*}{ Variables } & & Studies (N) & Sample (N) & \multicolumn{2}{|c|}{ Heterogeneity } & $95 \% \mathrm{Cl}$ & Pooled & \multicolumn{2}{|c|}{ Ratio of males to females } \\
\hline & & & & $1^{2}$ & $p$-value & & (mean) & $\begin{array}{l}\text { SMD } \mathbb{I}(95 \% \\
\mathrm{Cl})\end{array}$ & p-value \\
\hline \multirow{2}{*}{$\begin{array}{l}\text { Age at onset } \\
\text { of disease } \\
\text { (year) }\end{array}$} & Male & 10 & 3156 & 88.78 & $<0.001$ & $27.70-29.65$ & 28.67 & $\begin{array}{l}1.05(0.72, \\
1.37)\end{array}$ & $<0.001$ \\
\hline & Female & 9 & 8328 & 96.89 & $<0.001$ & $26.70-29.23$ & 27.96 & & \\
\hline \multirow[t]{2}{*}{ EDSS } & Male & 6 & 1219 & 97.71 & $<0.001$ & $2.76-4.20$ & 3.48 & $\begin{array}{l}0.30(-0.05 \\
0.67)\end{array}$ & 0.097 \\
\hline & Female & 6 & 2899 & 97.71 & $<0.001$ & $2.46-3.83$ & 3.14 & & \\
\hline \multicolumn{10}{|c|}{$\begin{array}{l}\text { †Number. } \\
\text { ‡Confidence interval. } \\
\text { \&Odds ratio. } \\
\text { I I Standardized mean difference. } \\
\text { EDSS: Expanded disability status s }\end{array}$} \\
\hline
\end{tabular}


Statistics for each study

\begin{tabular}{|c|c|c|c|c|c|c|}
\hline \multirow{2}{*}{$\frac{\text { Study name }}{\text { Saadatnia M (2007) }}$} & \multirow{2}{*}{$\begin{array}{c}\begin{array}{c}\text { Event } \\
\text { rate }\end{array} \\
0.122\end{array}$} & \multirow{2}{*}{$\begin{array}{c}\begin{array}{c}\text { Lower } \\
\text { limit }\end{array} \\
0.107\end{array}$} & \multirow{2}{*}{$\begin{array}{c}\begin{array}{c}\text { Upper } \\
\text { limit }\end{array} \\
0.138\end{array}$} & \multirow{2}{*}{$\begin{array}{c}\text { Z-value } \\
-26.786\end{array}$} & \multirow{2}{*}{$\begin{array}{c}\text { p-value } \\
0.000\end{array}$} & \multirow{2}{*}{$\begin{array}{c}\begin{array}{c}\text { Relative } \\
\text { weight }\end{array} \\
9.65\end{array}$} \\
\hline & & & & & & \\
\hline Hashemilar M (2011) & 0.071 & 0.057 & 0.089 & -20.884 & 0.000 & 9.21 \\
\hline Moghtaderi A (2012) & 0.062 & 0.036 & 0.104 & -9.403 & 0.000 & 6.98 \\
\hline Kalanie H (2003) & 0.050 & 0.027 & 0.090 & -9.075 & 0.000 & 6.48 \\
\hline Saman-Nezhad B (2012) & 0.012 & 0.005 & 0.028 & -10.165 & 0.000 & 5.09 \\
\hline Rezaali S (2013) & 0.112 & 0.089 & 0.140 & -15.887 & 0.000 & 9.13 \\
\hline Ashtari F (2011) & 0.201 & 0.171 & 0.235 & -13.468 & 0.000 & 9.42 \\
\hline Khoshravesh S (2016) & 0.105 & 0.086 & 0.128 & -18.580 & 0.000 & 9.29 \\
\hline Taraghi Z (2010) & 0.070 & 0.034 & 0.140 & -6.600 & 0.000 & 5.59 \\
\hline Lasemi E (2011) & 0.104 & 0.078 & 0.138 & -13.148 & 0.000 & 8.74 \\
\hline Danesh-Sani SA (2013) & 0.151 & 0.122 & 0.185 & -13.965 & 0.000 & 9.21 \\
\hline Taraghi Z (2007) & 0.070 & 0.034 & 0.139 & -6.633 & 0.000 & 5.62 \\
\hline \multirow[t]{2}{*}{ Pourmemari MH (2011) } & 0.073 & 0.035 & 0.145 & -6.478 & 0.000 & 5.59 \\
\hline & 0.089 & 0.069 & 0.114 & -16.595 & 0.000 & \\
\hline \multicolumn{6}{|c|}{ Heterogeneity: $I^{2}: 89.75 ; p<0.001$. Random effects model } & \\
\hline
\end{tabular}

Meta analysis

Figure 6. A positive family history of multiple sclerosis in Iranian multiple sclerosis patients.

Begg's tests was 0.447 and 0.488 , respectively), but was significant for MS family history (p-value for Egger and Begg's tests was 0.027 and 0.046, respectively) and clinical course of PR (p-value for Egger and Begg's tests was 0.003 and 0.363, respectively) (Supplementary Figure 2).

\section{Discussion}

The present study is the first systematic review and meta-analysis on published literature on MS clinical courses of RR, PP, SP and RP, age of disease onset, and positive family history in Iranian MS patients.

One of the main findings of this study, the RR course $(77.1 \%)$ was the most common form of MS disease in Iran, followed by PP (6.2\%), SP (9.5\%), and RP (0.4\%). The Meta regression model showed a reduction in the prevalence of RR clinical course and a increase in the prevalence of PP course in recent years but were not significant.

The findings are similar to those of other studies, indicating that the most common clinical form of clinical course are RR (between 33\% and 96.8\%), then PP (between 0.8\% and 34.3\%) and SP (2.4\% and 57\%) [59-68] and suggests that they are consistent with the results of this study.

The mean age of disease onset has been reported to be diverse in different studies: of 31.7 years in the study of TürkBörü [60], 30 years in the study of Ares [69], 34.6 years in the study of Bellantonio [62], 28.3 years in the study of Houzen [70] and 31.8 years in the study of Grytten [67]. In this study, the mean age of disease onset was 28.9 years.

In this study, the positive family history of MS were $8.9 \%$. In other studies, positive family history have been reported among relatives to be between $5 \%$ and $26 \%$ [71]. In a meta-analysis study in Iran, the prevalence of familial MS was reported 11.4\% (95\% CI: 8.7-14.1) [72]. In addition, in an Italian study, for different types of MS, there is an increase in the prevalence of first-degree relatives, especially among siblings, compared with children and parents [73]. In Europe, the incidence of familial MS ranges from the lowest (2\%) in Hungary to the highest (19\%) in England. It varies from 3\%-5\% in North America to $10.5 \%$ in Mexico to $75 \%-78 \%$ in Argentina [74-77]. In a case-control study in Iran, an estimate of the increased risk associated with MS among patients with a positive family history was more than eight times [78].

In 5374 MS patients, the mean score of EDSS was 2.83. In other studies, EDSS has been reported in patients with MS to be 1.6-6 [59,61,65]. The EDSS is the most popular Kurtzke tool [79]. EDSS is clinician administered to assess the CNS. EDSS is used to describe the progress of disease in MS patients and to assess the effectiveness of therapeutic interventions in clinical trials. This order system is rated from 0 (normal nervous state) to 10 (death 
due to MS) at an interval of 0.5 (when reaching EDSS 1). The lower scale values of the EDSS measure impairments based on the neurological examination, while the upper range of the scale (>EDSS 6) measures handicaps of patients with MS [80].

One limitation of this study is we not able to subgroup analysis based on Iranian subpopulation.

\section{Conclusion}

The results of this meta-analysis in Iranian population can provide useful information for neurologists and healthcare providers in the field of MS clinical courses, age of disease onset and disability in Iranian MS patients. This study showed that the most common clinical course in Iranian MS patients is RR. The prevalence of RR course during the studied years decreased and the prevalence of PP increased but were not significant.

\section{Summary points}

- The prevalence of clinical courses of relapsing remitting, primary progressive, secondary progressive and relapsing progressive in Iranian patients with MS was $77.1 \%$ (95\% Cl: $72.2-81.3), 6.2 \%$ (95\% Cl: 4.1-9.2), 9.5\% (95\% Cl: 6.8-13.1) and $0.4 \%(95 \% \mathrm{Cl}: 0.2-0.8)$, respectively. This study showed that the most common clinical course in Iranian MS patients is RR.

- The prevalence of RR course during the studied years decreased and the prevalence of primary progressive increased but were not significant.

- Positive family history of MS in Iranian patients with MS was 8.9\% (95\% Cl: 6.9-11.4).

- The mean age of disease onset of MS was 28.9 years (95\% Cl: 27.8-30.1).

- The results of this meta-analysis can provide useful information for neurologists and healthcare providers in the field of MS clinical course, age of disease onset and disability in Iranian MS patients.

\section{Financial \& competing interests disclosure}

The authors have no relevant affiliations or financial involvement with any organization or entity with a financial interest in or financial conflict with the subject matter or materials discussed in the manuscript. This includes employment, consultancies, honoraria, stock ownership or options, expert testimony, grants or patents received or pending, or royalties.

No writing assistance was utilized in the production of this manuscript.

\section{Open access}

This work is licensed under the Attribution-NonCommercial-NoDerivatives 4.0 Unported License. To view a copy of this license, visit http://creativecommons.org/licenses/by-nc-nd/4.0/

\section{References}

Papers of special note have been highlighted as: $\bullet$ of interest; $\bullet \bullet$ of considerable interest

1. Browne P, Chandraratna D, Angood C et al. Atlas of multiple sclerosis 2013: a growing global problem with widespread inequity. Neurology 83(11), 1022-1024 (2014).

2. Kargarfard M, Eetemadifar M, Mehrabi $M$ et al. Fatigue, depression, and health-related quality of life in patients with multiple sclerosis in Isfahan, Iran. Eur. J. Neurol. 19(3), 431-443 (2012).

3. Opara J, Jaracz K, Brola W. Burden and quality of life in caregivers of persons with multiple sclerosis. Neurol. Neurochir. Pol. 46(5), 472-479 (2012).

4. Veauthier $\mathrm{C}$, Radbruch $\mathrm{H}$, Gaede $\mathrm{G}$ et al. Fatigue in multiple sclerosis is closely related to sleep disorders: a polysomnographic cross-sectional study. Mult. Scler. 17, 613-622 (2011).

5. Riley CS, Tullman MJ. Multiple Sclerosis. 12th Edition. Wolters Kluwer/Lippincott Williams \& Wilkins, PA, USA, $903-918$ (2010).

6. Weinges-Evers N, Brandt A, Bock M et al. Correlation of self-assessed fatigue and alertness in multiple sclerosis. Mult. Scler. 16, 1134-1140 (2010).

7. Heydarpour P, Mohammad K, Yekaninejad MS et al. Multiple sclerosis in Tehran, Iran: a joinpoint trend analysis. Mult. Scler. 20,512 (2014).

8. Etemadifar M, Abtahi S-H, Akbari M et al. Multiple sclerosis in Isfahan, Iran: an update. Mult. Scler. 20, 1145-1147 (2013).

9. Alroughani R, Ahmed SF, Behbahani R et al. Increasing prevalence and incidence rates of multiple sclerosis in Kuwait. Mult. Scler. 20, 543-547 (2014).

10. Alroughani R et al. Increasing prevalence and incidence rates of multiple sclerosis in Kuwait. Mult. Scler. 20, 543-547 (2014). 
11. Deleu D, Mir D, Al Tabouki A et al. Prevalence, demographics and clinical characteristics of multiple sclerosis in Qatar. Mult. Scler. 19(6), 816-819 (2013).

12. Kurtzke JF. Epidemiologic contributions to multiple sclerosis: an overview. Neurology 30(7 Pt 2), 61-79 (1980).

13. Kurtzke JF. A reassessment of the distribution of multiple sclerosis. Acta. Neurol. Scand. 51(2), 137-157 (1975).

14. Azami M, Yekta Kooshali MH, Shohani M, Khorshidi A, Mahmudi L. Epidemiology of multiple sclerosis in Iran: A systematic review and meta-analysis. PLoS ONE 14(4), e0214738 (2019).

15. Izadi S, Nikseresht AR, Poursadeghfard M et al. Prevalence and incidence of multiple sclerosis in Fars Province, Southern Iran. Iran. J. Med. Sci. 40(5), 390 (2015).

16. Ashjazadeh N, Hadianfard H, Feridoni $S$ et al. Assessment of health-related quality of life in patients with multiple sclerosis living in the Fars province of Iran. Neuroimmunol. Neuroinflammation 3, 57-62 (2016).

17. Izadi S, Nikseresht A, Poursadeghfard M. Epidemiology of multiple sclerosis in Fars Province. irje 10(2), 56-61 (2014).

18. Jajvandian R, Ali Babai A, Torabzadeh S et al. Prevalence of multiple sclerosis in North Khorasan province, northern Iran; in 5th Joint Triennial Congress of the European and Americas Committees for Treatment and Research in Multiple Sclerosis. Amsterdam, The Netherlands. Mult. Scler. 17(Suppl. 10), S9-524 (2011).

19. Sayehmiri K, Tavan H, Sayehmire F, et al. Prevalence of epilepsy in Iran using meta-analysis and systematic review. ZUMS J. 23(97), 112-121 (2015)

20. Sayehmiri K, Tavan H. Systematic review and meta-analysis methodsprevalence of peptic ulcer in Iran. Govaresh 20(4), 250-258 (2016).

21. Shamseer L, Moher D, Clarke M et al. Eds. PRISMA-P Group. Preferred reporting items for systematic review and meta-analysis protocols (PRISMA-P) 2015: elaboration and explanation. BMJ 349, g7647 (2015).

22. Wells GA, Shea B, O'Connell D et al. The Newcastle-Ottawa Scale (NOS) for assessing the quality of nonrandomized studies in meta-analyses. (2011) http://www.ohri.ca/programs/clinical_epidemiology/oxford.asp

23. Ades AE, Lu G, Higgins JP. The interpretation of random-effects meta-analysis in decision models. Med. Decis. Making 25(6), 646-654 (2005).

24. Majdinasab N, Nakhostin-Mortazavi A, Alemzadeh-Ansari MH. Epidemiologic features of multiple sclerosis in south-western Iran; in 28th Congress of the European Committee for Treatment and Research in Multiple Sclerosis. Lyon, France, 2012. Mult. Scler. 18(Suppl. 4), 9-542 (2012)

25. Etemadifar M, Maghzi AH. Sharp increase in the incidence and prevalence of multiple sclerosis in Isfahan, Iran. Mult. Scler. 17, 1022-1027 (2011).

26. Dehghan-Nayeria N, Khakbazan Z, Ghafoori F et al. Sexual dysfunction levels in Iranian women suffering from multiple sclerosis. Mult. Scler. Relat. Disord. 12, 49-53 (2017).

27. Choobforouszade A, Neshatdoos $\mathrm{H}$, Molavi $\mathrm{H}$ et al. Examine the quality of life among Multiple sclerosis patients in Yazd MS Association, Iran. J. Tolooebehdasht 14(5), 131-140 (2016).

28. Saman-Nezhad B, Rezaee T, Bostani A et al. Epidemiological characteristics of patients with multiple sclerosis in Kermanshah, Iran in 2012. J. Mazandaran. Univ. Med. Sci. 23(104), 97-101 (2013).

29. Moghtaderi A, Rakhshanizadeh F, Shahrakilbrahimi S. Incidence and prevalence of multiple sclerosis in southeastern Iran. Clin. Neurol. Neurosurg. 115, 304-308 (2013).

30. Taraghi Z, Ilali E, Abedini M et al. Quality of life among multiple sclerosis patients. Iran J. Nursing 20(50), 51-59 (2007).

31. Pourmemari MH, Rabiei S, Bagheri $\mathrm{H}$ et al. Epidemiologic variables in multiple sclerosis patients in Zanjan. Holistic. Nursing Midwifery 21(1), 1-6, 11 (2011).

32. Lasemi E, Sahraian MA, Kalantar Motamedi MH et al. Prevalence of oral and maxillofacial manifestations in patients with multiple sclerosis. J. Res. Dent. Sci. 8(1), 20-26 (2011).

33. Khoshravesh S, Taymoori P, Abdolmaleki $S$ et al. Awareness, risk perception, and protective behaviors in regard to multiple sclerosis among people in Sanandaj, Iran. Sci. J. Kurdistan. Univ. Med. Sci. 21(1), 10-20 (2016).

34. Danesh-Sani SA, Rahimdoost A, Soltani M et al. Clinical assessment of orofacial manifestations in 500 patients with multiple sclerosis. J. Oral. Maxillofac. Surg. 71(2), 290-294 (2013).

35. Ashtari F, Shaygannejad V, Heidari F et al. Prevalence of familial multiple sclerosis in Isfahan, Iran. J. Isfahan Med. Sch. 29(138), 551-561 (2011).

36. Hojjati SMM, Zarghami A, Hojjati SA et al. Optic neuritis, the most common initial presenting manifestation of multiple sclerosis in northern Iran. Caspian. J. Intern. Med. 6(3), 151 (2015).

37. Saadat SMS, Hosseininezhad M, Bakhshayesh B et al. Prevalence and predictors of depression in Iranian patients with multiple sclerosis: a population-based study. Neurol. Sci. 35(5), 735-740 (2014).

38. Rezapoor A, Almasian KA, Goodarzi S et al. The assessment of disease characteristics on multiple sclerosis patients' quality of life in Iran. Epidemiol. Health. 19, 39, e2017008 (2017). 
39. Torabipour A, Asl ZA, Majdinasab $\mathrm{N}$ et al. A study on the direct and indirect costs of multiple sclerosis based on EDSS score in Khuzestan, Iran. Int. J. Prev. Med. 5(9), 555-561 (2014).

40. Alehashemi A, Nourian A, Maleki M et al. Frequency of primary headache in patients with multiple sclerosis in Mashhad. Shefaye. Khatam. 3(2), 55-60 (2015).

41. Taraghi Z, Ilali E. Quality of life among multiple sclerosis patients. J. Applied. Sci. 10(14), 1485-1488 (2010).

42. Karampoor S, Zahednasab H, Ramagopalan S et al. 25-hydroxyvitamin D levels are associated with multiple sclerosis in Iran: a cross-sectional study. J. Neuroimmunol. 290, 47-48 (2016).

43. Ayatollahi P, Nafissi S, Eshraghian M et al. Impact of depression and disability on quality of life in Iranian patients with multiple sclerosis. Mult. Scler. 13, 275-277 (2007).

44. Ghajarzadeh M, Sahraian MA, Fateh R et al. Fatigue, depression and sleep disturbances in Iranian patients with multiple sclerosis. Acta. Medica. Iranica. 50(4), 244 (2012).

45. Nabavi SM, Poorfarzam Sh, Ghassemi H. Clinical course and prognosis of 203 patients with MS in Shahid Mostafa Khomeini Hospital, Tehran 2002. Tehran. Univ. Med. J. 64(7), 90-97 (2006).

46. Mohammadi K, Rahnama P, Mohseni SM et al. Determinants of sexual dysfunction in women with multiple sclerosis. $B M C$ Neurology 13(1), 83 (2013).

47. Saberi A, Roudbary SA, Hatamian HR et al. Assessing the frequency of different types of multiple sclerosis in MS patients with \& without seizure in Guilan Province. J. Mazandaran Univ. Med. Sci. 21(1), 235-42 (2012).

48. Sahraian MA, Khorramnia S, Ebrahim MM et al. Multiple sclerosis in Iran: a demographic study of 8,000 patients and changes over time. Eur. Neurol. 64, 331-336 (2010).

49. Saadatnia M, Etemadifar M, Maghzi AH. Multiple sclerosis in Isfahan, Iran. Int. Rev. Neurobiol. 79, 357-375 (2007).

50. Khanjani Z. Effective psychological factors on multiple sclerosis: personality traits, depression, anxiety and stress. Tabriz. Uni. Med. Sci. 34(4), 60-67 (2012).

51. Kargarfard M, Eetemadifar M, Mehrabi M et al. Fatigue, depression, and health-related quality of life in patients with multiple sclerosis in Isfahan, Iran. Eur. J. Neurol. 19(3), 431-437 (2012).

52. Abedini M, Habibi-Saravi R, Zarvani A, Farahmand M. Epidemiology of multiple sclerosis in Mazandran province in 2007. J. Mazandaran Univ. Med. Sci. 18, 82-87 (2008).

53. Kalanie H, Gharagozli K, Kalanie AR. Multiple sclerosis: report on 200 cases from Iran. Mult. Scler. 9, 36-38 (2003).

54. Elhami SR, Mohammad K, Sahraian MA et al. A 20-year incidence trend (1989-2008) and point prevalence (March 20, 2009) of multiple sclerosis in Tehran, Iran: a population-based study. Neuroepidemiology 36, 41-147 (2011).

55. Hashemilar M, Savadi Ouskui D, Farhoudi M et al. Multiple sclerosis in east Azerbaijan, North West Iran. Neurol. Asia 16, 127-131 (2011)

56. Ebrahimi HA, Sedighi B. Prevalence of multiple sclerosis and environmental factors in Kerman province, Iran. Neurol. Asia 18, 385-389 (2013).

57. Asgari A, Mehrabi F. Epidemiology of multiple sclerosis (MS) in military personnel: demographic study in Iran. Biomed. Pharmacol. J. 8 (March Spl. Edition), 105-110 (2015).

58. Rezaali S, Khalilnezhad A, Moghadasi AN et al. Epidemiology of multiple sclerosis in Qom: demographic study in Iran. Iran. J. Neurol. 12(4), 136-143 (2013).

59. Cattaneo D, Lamers I, Bertoni R et al. Participation restriction in people with multiple sclerosis: prevalence and correlations with cognitive, walking, balance, and upper limb impairments. Arch. Phys. Med. Rehabil. 98(7), 1308-1315 (2017).

60. Türk Börü U, Alp R, Sur H, Gül L. Prevalence of multiple sclerosis door-to-door survey in Maltepe, Istanbul, Turkey. Neuroepidemiology 27(1), 17-21 (2006).

61. Coskun Benlidayi I, Basaran S, Evlice A, Erdem M, Demirkiran M. Prevalence and risk factors of low bone mineral density in patients with multiple sclerosis. Acta. Clinica. Belgica. 70(3), 188-192 (2015).

62. Bellantonio P, Iuliano G, Di Blasio F, Ruggieri S. Prevalence and incidence of multiple sclerosis in Campobasso (Molise region chieftown, southern Italy). Clin. Neurol. Neurosurg. 115(9), 1806-1808 (2013).

63. Niino M, Miyazaki Y. Cognitive impairment in multiple sclerosis. Brain Nerve 68(4), 375-381 (2016).

64. Simonsen CS, Edland A, Berg-Hansen P et al. High prevalence and increasing incidence of multiple sclerosis in the Norwegian county of Buskerud. Acta Neurol. Scand. 135(4), 412-418 (2017).

65. Heitmann H, Biberacher V, Tiemann L et al. Prevalence of neuropathic pain in early multiple sclerosis. Mult. Scler. 22(9), 1224-1230 (2016).

66. Guimond C, Lee JD, Ramagopalan SV et al. Multiple sclerosis in the Iranian immigrant population of BC, Canada: prevalence and risk factors. Mult. Scler. 20(9), 1182-1188 (2014). 
67. Grytten N, Aarseth JH, Lunde HM et al. A 60-year follow-up of the incidence and prevalence of multiple sclerosis in Hordaland County, Western Norway. J. Neurol. Neurosurg. Psychiatry. 87(1), 100-105 (2016).

68. Gracia F, Castillo LC, Benzadon A et al. Prevalence and incidence of multiple sclerosis in Panama (2000-2005). Neuroepidemiology 32(4), 287-293 (2009).

69. Ares B, Prieto JM, Lema M et al. Prevalence of multiple sclerosis in Santiago de Compostela (Galicia, Spain). Mult. Scler. 13(2), 262-264 (2007).

70. Houzen $\mathrm{H}$, Niino M, Hirotani $\mathrm{M}$ et al. Increased prevalence, incidence, and female predominance of multiple sclerosis in northern Japan. J. Neurol. Sci. 323(1-2), 117-122 (2012).

71. Cabrera-Gomez JA, Ramon-Perez L, Saiz A et al. Neuromyelitis optica and multiple sclerosis in sisters. Mult. Scler. 15(2), 269-271 (2009).

72. Moosazadeh M, Esmaeili R, Mehdi Nasehi M et al. Prevalence of familial multiple sclerosis in Iran: a systematic review and meta-analysis. Iran. J. Neurol. 16(2), 90-95 (2017).

73. Guaschino C, Esposito F, Liberatore G et al. Familial clustering in Italian progressive-onset and bout-onset multiple sclerosis. Neurol. Sci. 35(5), 789-791 (2014).

74. Fricska-Nagy Z, Bencsik K, Rajda C et al. Epidemiology of familial multiple sclerosis in Hungary. Mult. Scler. 13(2), 260-261 (2007).

75. Melcon MO, Correale J, Melcon CM. Is it time for a new global classification of multiple sclerosis? J. Neurol. Sci. 344(1-2), 171-181 (2014).

76. Farez MF, Balbuena Aguirre ME, Varela F et al. Low familial risks for multiple sclerosis in Buenos Aires, Argentina. J. Neurol. Sci. 346(1-2), 268-270 (2014).

77. Gonzalez O, Sotelo J. Is the frequency of multiple sclerosis increasing in Mexico? J. Neurol. Neurosurg. Psychiatry 59(5), 528-530 (1995).

78. Eskandarieh S, Nedjat S, Abdollahpour I et al. Comparing epidemiology and baseline characteristic of multiple sclerosis and neuromyelitis optica: a case-control study. Mult. Scler. Relat. Disord. 12, 39-43 (2017).

79. Kurtzke JF. Rating neurologic impairment in multiple sclerosis: an Expanded Disability Status Scale (EDSS). Neurology 33(11), 1444-1452 (1983).

80. Ineichen BV, Schneider MP, Hlavica M et al. High EDSS can predict risk for upper urinary tract damage in patients with multiple sclerosis. Mult. Scler. 24(4), 529-534 (2017).

81. Richardson WS, Wilson MC, Nishikawa J et al. The well-built clinical question: a key to evidence-based decisions. ACP J. Club 123 , A12-3 (1995). 
